(2) Open Access Full Text Article

CASE REPORT

\title{
Histopathologic correlation of Aspergillus endophthalmitis following uncomplicated cataract
} surgery

This article was published in the following Dove Press journal:

Clinical Ophthalmology

24 September 2012

Number of times this article has been viewed

\section{Luis J Haddock' \\ Harry W Flynn Jr' \\ Sander R Dubovy' \\ Rahul N Khurana ${ }^{2}$ \\ Peter R Egbert ${ }^{3}$ \\ 'Department of Ophthalmology, Bascom Palmer Eye Institute, Miami Miller School of Medicine, Miami, FL, ${ }^{2}$ Northern California Retina Vitreous Associates, Mountain View, CA, ${ }^{3}$ Department of Ophthalmology, The Byers Eye Institute at Stanford, Stanford University, Palo Alto, CA, USA}

Correspondence: Luis J Haddock Bascom Palmer Eye Institute, 900 NW 17th Street, Miami, FL 33136, USA

Tel +I 3053266000

$\mathrm{Fax}+\mathrm{I} 3053266417$

Email Ihaddock@med.miami.edu
Abstract: A clinicopathologic correlation between two patients with acute-onset Aspergillus endophthalmitis undergoing enucleation is reported. These two patients presented with pain, redness, and decreased vision following uncomplicated cataract surgery. In both patients, vitreous aspiration and intravitreal injections were the initial treatment followed later by pars plana vitrectomy for clinical worsening. Despite repeated surgical and medical interventions, the clinical course of both patients was prolonged, unsuccessful, and resulted in enucleation for a blind painful eye. Histologic examination of the enucleated specimens showed that, in spite of prolonged local and systemic therapy, there was persistent diffuse infiltration of the anterior chamber and ciliary body by a filamentous mold.

Keywords: mold, enucleation, voriconazole, amphotericin B

\section{Introduction}

Acute-onset endophthalmitis after cataract surgery is rarely caused by fungus in Western nations. Gram-positive bacteria such as coagulase-negative Staphylococcus cause most cases of postoperative endophthalmitis. ${ }^{1}$ Most cases of fungal endophthalmitis have an endogenous source and occur in patients with history of immunosuppression or intravenous drug use. Cases of exogenous fungal endophthalmitis are less common, and have been found to be secondary to keratitis, trauma, or rarely postoperative. In a large review of fungal endophthalmitis, the most common isolate following intraocular surgery was Aspergillus. ${ }^{2}$ Patients with Aspergillus endophthalmitis have been found to have a complicated clinical course with poor visual outcomes, often resulting in enucleation. ${ }^{2}$ We report two patients with acute-onset endophthalmitis after uncomplicated cataract extraction caused by Aspergillus who had persistent intraocular fungal organisms at the time of enucleation despite extensive surgical and medical management.

\section{Case report I}

A 69-year-old female without any prior ocular history or relevant systemic medical problems presented with pain and decreased visual acuity to 20/70 in the right eye 16 days after uncomplicated cataract surgery. The cataract surgery was performed using phacoemulsification with a lens placed in the bag and no capsular rupture was encountered. After examination on presentation, a diagnosis of acute-onset endophthalmitis was made, and vitreous aspiration and intravitreal injections of vancomycin $1 \mathrm{mg} / 0.1 \mathrm{~mL}$, ceftazidime $2.25 \mathrm{mg} / 0.1 \mathrm{~mL}$, and dexamethasone $0.4 \mathrm{mg} / 0.1 \mathrm{~mL}$ were performed. The following day, visual acuity decreased to $6 / 200$ and a pars plana vitrectomy was performed with repeat 
injections (vancomycin, ceftazidime, dexamethasone). Initial cultures revealed Aspergillus fumigatus for which intravitreal amphotericin B $5 \mu \mathrm{g} / 0.1 \mathrm{~mL}$ was given twice. Two weeks later, visual acuity decreased to light perception, and pars plana vitrectomy, lens removal, and repeat amphotericin B injection was performed. The patient was referred to Bascom Palmer Eye Institute 1.5 months after presentation with light perception
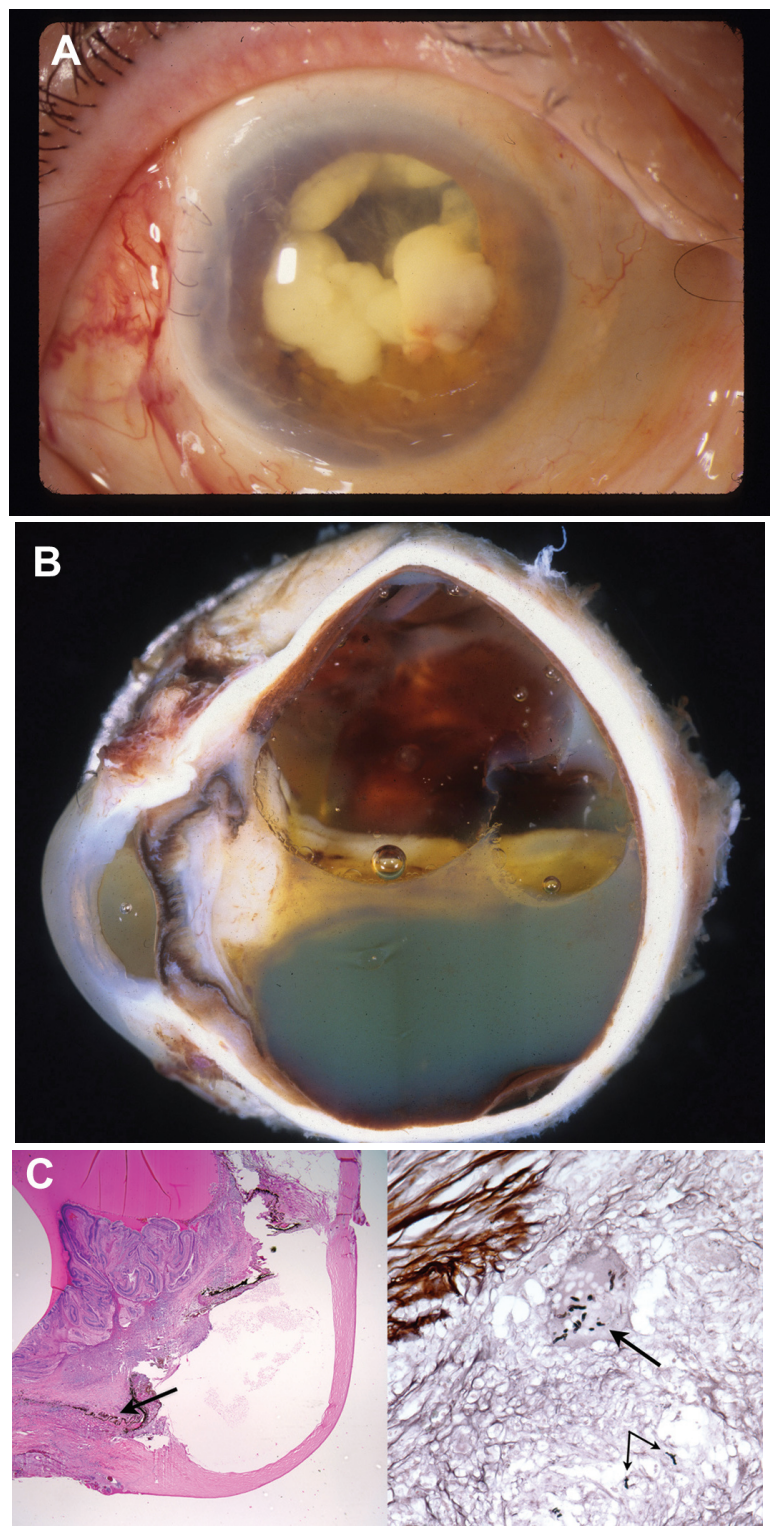

Figure I (A) Slit lamp photograph of case I at presentation displaying fungal masses in the anterior chamber, representing a large Aspergillus infiltrate. (B) Gross examination of the globe in case I discloses a funnel-shaped retinal detachment, a vitreous cavity full of silicone oil, a closed anterior chamber with a dense cyclitic membrane, and a white fibrous plaque on the anterior and posterior surface of the iris. (C) Left image shows low magnification histopathology of the anterior segment of case I, and is magnified on the right image.

Notes: On this image, examination of the ciliary body of case I displays septated hyphae consistent with Aspergillus. Cultures confirmed this organism. (Left, hematoxylin-eosin staining, original magnification $\times 100$; right, Gomori methamine silver staining, original magnification $\times 400$ ). vision and an examination remarkable for white fluffy opacities in the anterior chamber (Figure 1A). Ultrasound revealed a retinal detachment. Repeat vitrectomy was performed to repair the retinal detachment, with placement of silicone oil and injection of voriconazole $200 \mu \mathrm{g}$. Subsequent cultures were negative. Despite aggressive surgical (three pars plana vitrectomies and lens removal) and medical (three intravitreal injections of amphotericin B and one of voriconazole) management, enucleation was performed for a blind painful eye.

\section{Case report 2}

A 56-year-old female without any prior ocular history or relevant systemic medical problems presented with pain and visual acuity decreased to 20/70 in the right eye 14 days after uncomplicated cataract surgery. The cataract surgery was performed using phacoemulsification without capsule rupture and with a lens placed in the bag. Examination on presentation was remarkable for a large fibrin plaque in the anterior chamber and dense vitritis. After a diagnosis of acute-onset endophthalmitis was made, vitreous aspiration and intravitreal injections of vancomycin $1 \mathrm{mg} / 0.1 \mathrm{~mL}$ and ceftazidime $2.25 \mathrm{mg} / 0.1 \mathrm{~mL}$ were performed. Two days later, visual acuity decreased to hand motion and the treating physician elected not to proceed with vitrectomy because of reducing pain and decreased conjunctival congestion, and a second tap and inject (vancomycin and ceftazidime) was performed. Both initial cultures were negative. One month later, the patient was referred to Bascom Palmer Eye Institute with light perception vision, and examination was remarkable for complete hypopyon, corneal edema, a white plaque over the iris, and moderately dense vitreous membranes by ultrasound (Figure 2A). The patient was treated with vitrectomy, lens removal, and repeat injections for persistent infection. Cultures were positive for A. fumigatus. The patient received intravitreal amphotericin B $5 \mu \mathrm{g} / 0.1 \mathrm{~mL}$ followed by voriconazole $100 \mu \mathrm{g} / 0.1 \mathrm{~mL}$, and was started on oral voriconazole $200 \mathrm{mg}$ orally twice daily. The patient later developed a retinal detachment that was repaired with pars plana vitrectomy and placement of silicone oil. Subsequent cultures were all negative. Despite extensive surgical and medical management, enucleation was performed for a blind painful eye.

The histopathology of both enucleated specimens displaying fungal elements within the anterior chamber and anterior vitreous is described in Figures 1B, C and 2B, C.

\section{Discussion}

Aspergillus species are ubiquitous molds found in organic matter, with more than 100 species identified. The majority 


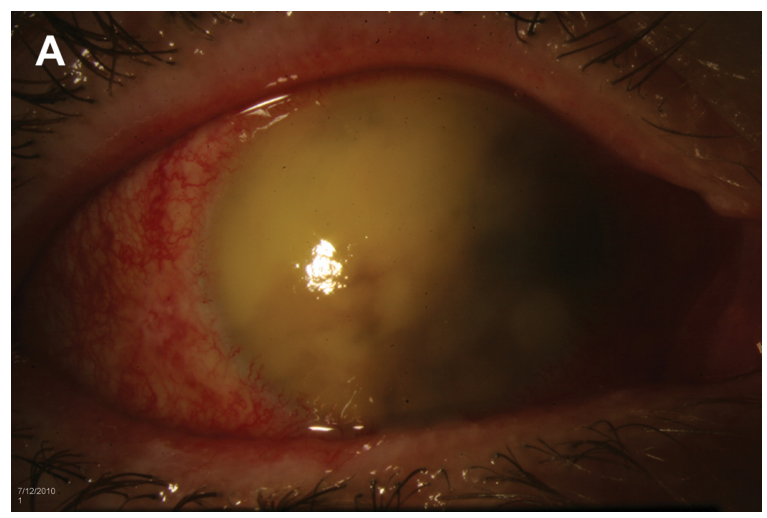

\section{B}
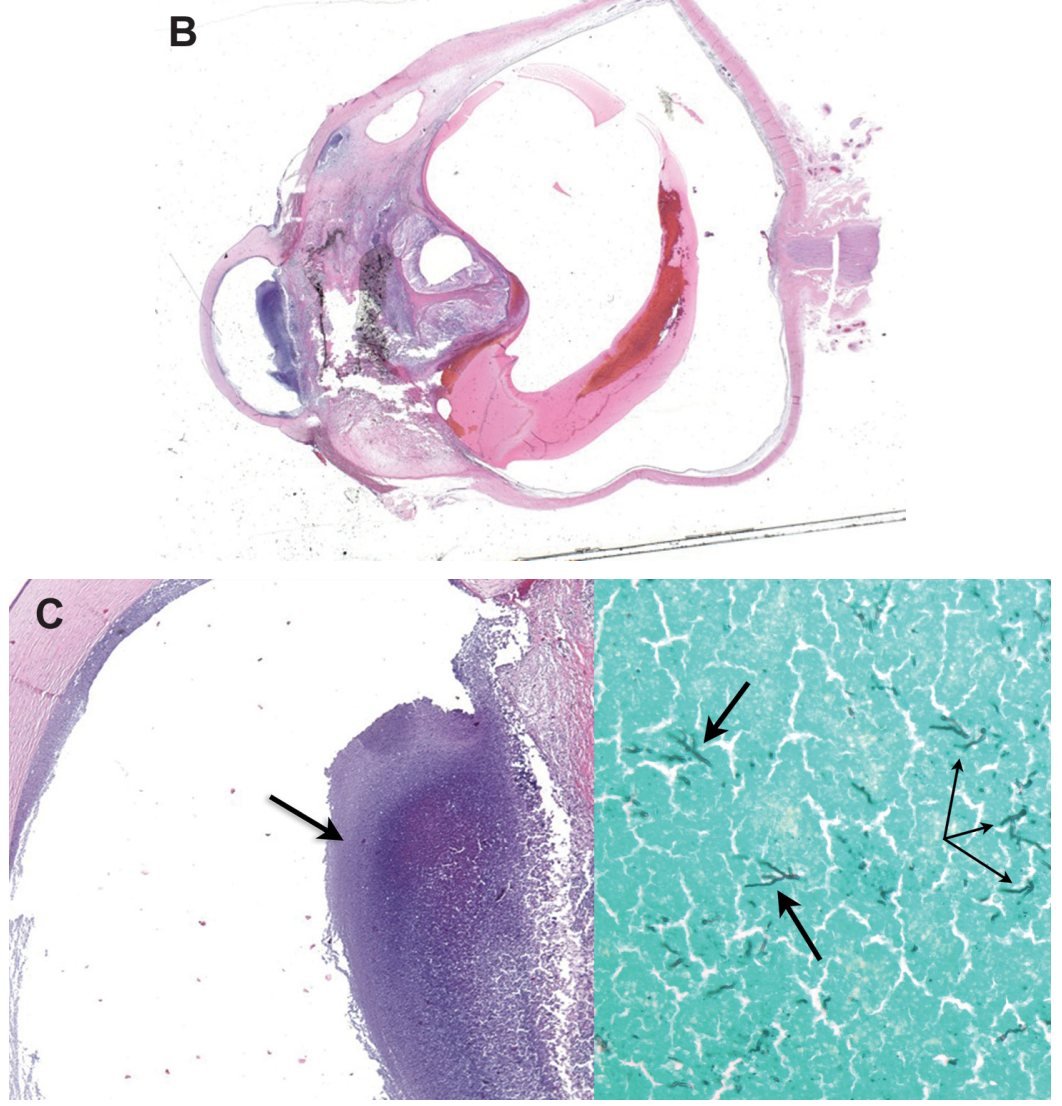

Figure 2 (A) Slit lamp photograph of case 2 at presentation showing corneal edema, and a complete hypopyon with dense fibrin within the anterior chamber. (B) Histopathology of case 2 displays a centrally gathered and detached retina with inflammatory material present within the anterior chamber and anterior vitreous. (Hematoxylin-eosin staining, original magnification $\times 40$ ). (C) Histopathology on the left indicating area of collection in the anterior chamber, highlighted in the image on the right showing the contents of the anterior chamber displaying septated fungal hyphae consistent with Aspergillus.

Notes: Cultures confirmed this organism. (Left, hematoxylin-eosin staining, original magnification $\times 100$; right, Gomori methamine silver staining, original magnification $\times 400$ ).

of human illness is caused by A. fumigatus. ${ }^{3}$ Cases of exogenous Aspergillus endophthalmitis are rare, but may be seen in immunocompetent patients secondary to trauma, keratitis, or following intraocular surgery. ${ }^{4,5}$ Inoculation of the organism during intraocular surgery, such as cataract surgery, results in an acute-onset postoperative endophthalmitis that mimics bacterial endophthalmitis, often leading to a delay in diagnosis and management. ${ }^{6}$ Previous papers have reported a delay in the presentation of fungal endophthalmitis after cataract surgery; however, the two patients reported here presented within the first two weeks after cataract surgery, suggesting the possibility of a high load of fungal inoculum during surgery or in the early postoperative period. ${ }^{2,7,8}$ Although both patients presented early in the postoperative period, their initial management was different because the first patient had initial cultures revealing Aspergillus for which antifungal treatment was started early in the disease course, and the second patient had initial cultures which 
were negative, and antifungal treatment was delayed until cultures were positive. These two patients represent two problems which are commonly encountered when treating patients with this disease, ie, clinical worsening despite appropriate early treatment, and delay in treatment because of initial negative culture results. The histologic finding of viable fungal organisms after enucleation shows that this infection can be very aggressive and resistant to treatment. No risk factors for the development of fungal infections were identified in either patient. These two infections were isolated events occurring in different geographic locations (case 1 in Tampa, FL, and case 2 in San Francisco, CA).

The visual acuity outcomes of Aspergillus endophthalmitis are generally poor but highly variable. In a large study of exogenous fungal endophthalmitis, 13 of 41 cases occurred after intraocular surgery, with five of these cases caused by Aspergillus. Final visual acuity was 20/400 or better in $77 \%$ of patients, while $8 \%$ had no light perception. ${ }^{2}$ A series from India reported on 27 patients with exogenous fungal endophthalmitis after cataract surgery over four years, with 20 (74\%) of these cases positive for Aspergillus. Five of these patients achieved a final visual acuity better than 20/200, while nine had no light perception and three underwent enucleation. ${ }^{9}$ Callanan et al reported on a series of five patients with endophthalmitis after cataract surgery caused by Aspergillus, with three of the cases resulting in enucleation and one case achieving a final visual acuity better than 20/200. ${ }^{6}$ Furthermore, a large review of acuteonset endophthalmitis after clear corneal cataract surgery found that endophthalmitis caused by an organism other than coagulase-negative Staphylococcus was a risk factor for poor visual outcome. ${ }^{10}$ Similar to these studies, the two patients in the current report had poor outcomes because of the aggressive nature of this fungus and difficulty in identifying it and treating it successfully.

Like bacterial endophthalmitis, patients with fungal endophthalmitis can present with decreased vision, pain, hypopyon, and blurring of the iris details. Initially, the infection is limited to the anterior segment, but later spreads to involve the entire eye. Both of the patients reported presented with a persistent white plaque in the anterior chamber thought to be a focal area of a high concentration of fungal organisms. ${ }^{5}$ Fungal organisms were identified histologically, correlating with this area of the anterior segment. In addition, we noted that as the infection spread to the posterior segment and the inflammatory response became more prominent, both patients developed retinal detachments that needed surgical intervention. Interestingly, fungal organisms were not identified histologically in the posterior segment, suggesting a possible antimicrobial effect of silicone oil after surgery. In fact, previous reports have confirmed that using silicone oil in these complicated cases is appropriate because it does not support the growth of microbes, including both bacterial and fungal isolates. ${ }^{11}$ Furthermore, it helps reduce rates of subsequent retinal detachments and may help stabilize progression of hypotony. ${ }^{12}$ Use of initial steroids in the first patient did not improve the clinical course and may have been associated with worsening of the infection.

In the current report, the severe inflammation caused by Aspergillus may have contributed to the development of complications, including retinal detachment, cyclitic membrane formation, and subsequent contraction of fibrous tissue. The exact cause of the subsequent retinal detachment in this patient is uncertain, but potential causes may include the performing of a previous pars plana vitrectomy, the severe infectious involvement of the vitreous, and possible preexisting retinal abnormalities. The presence of fungal organisms in the anterior segment of the enucleated specimens demonstrates that Aspergillus may be present within the eye despite repeated treatment with intravitreal injections of amphotericin B or voriconazole, as well as vitrectomy.

\section{Disclosure}

The authors report no financial interest or any other conflicts of interest in this work.

\section{References}

1. Endophthalmitis Vitrectomy Study Group. Results of the Endophthalmitis Vitrectomy Study. A randomized trial of immediate vitrectomy and of intravenous antibiotics for the treatment of postoperative bacterial endophthalmitis. Arch Ophthalmol. 1995;113:1479-1496.

2. Wykoff CC, Flynn HW Jr, Miller D, Scott IU, Alfonso EC. Exogenous fungal endophthalmitis: microbiology and clinical outcomes. Ophthalmology. 2008;115(9):1501-1507.

3. Batra V, Steele R. Aspergillosis. Available from: http://emedicine.medscape.com/article/960938-overview. Accessed August 22, 2012.

4. Pflugfelder SC, Flynn HW Jr, Zwickey TA, et al. Exogenous fungal endophthalmitis. Ophthalmology. 1988;95(1):19-30.

5. Alliman KJ, De Almeida FP, Flynn HW, Vanderveldt SL. Recurrent endophthalmitis following cataract surgery. Retina Physician. 2010;6: 56-58.

6. Callanan D, Scott IU, Murray TG, Oxford KW, Bowman CB, Flynn HW Jr. Early onset endophthalmitis caused by Aspergillus species following cataract surgery. Am J Ophthalmol. 2006;142(3):509-511.

7. Theodre FH. Mycotic endophthalmitis after cataract surgery. Int Ophthalmol Clin. 1961;4:861-887.

8. Fine BS, Zimmerman LE, Richards AB. Exogenous intraocular fungal infections with particular reference to complications of intraocular surgery. Am J Ophthalmol. 1959;48:151-165.

9. Narang S, Gupta A, Gupta V, et al. Fungal endophthalmitis following cataract surgery: clinical presentation, microbiological spectrum, and outcome. Am J Ophthalmol. 2001;132(5):609-617. 
10. Lalwani GA, Flynn HW Jr, Scott IU, et al. Acute-onset endophthalmitis after clear corneal cataract surgery (1996-2005). Clinical features, causative organisms, and visual acuity outcomes. Ophthalmology. 2008;115(3):473-476.

11. Chrapek O, Vecerova R, Koukalova D, et al. The in vitro antimicrobial activity of silicone oils used in ophthalmic surgery. Biomed Pap Med Fac Univ Palacky Olomouc Czech Repub. 2012;156(1):7-13.
12. Aras C, Ozdamar A, Karacorlu M, Ozkan S. Silicone oil in the surgical treatment of endophthalmitis associated with retinal detachment. Int Ophthalmol. 2001;24(3):147-150.

\section{Publish your work in this journal}

Clinical Ophthalmology is an international, peer-reviewed journal covering all subspecialties within ophthalmology. Key topics include: Optometry; Visual science; Pharmacology and drug therapy in eye diseases; Basic Sciences; Primary and Secondary eye care; Patien Safety and Quality of Care Improvements. This journal is indexed on

Submit your manuscript here: http://www.dovepress.com/clinical-ophthalmology-journal

\section{Dovepress}

PubMed Central and CAS, and is the official journal of The Society of Clinical Ophthalmology (SCO). The manuscript management system is completely online and includes a very quick and fair peer-review system, which is all easy to use. Visit http://www.dovepress.com/ testimonials.php to read real quotes from published authors. 